\title{
Quantitative multichannel NC-AFM data analysis of graphene growth on $\mathrm{SiC}(0001)$
}

\author{
Christian Held ${ }^{1}$, Thomas Seyller ${ }^{2}$ and Roland Bennewitz ${ }^{* 1}$
}

\author{
Full Research Paper \\ Address: \\ ${ }^{1}$ INM - Leibniz-Institute for New Materials, Campus D2 2, 66123 \\ Saarbrücken, Germany and ${ }^{2}$ Lehrstuhl für Technische Physik, \\ Universität Erlangen-Nürnberg, 91058 Erlangen, Germany \\ Email: \\ Roland Bennewitz ${ }^{*}$ - roland.bennewitz@inm-gmbh.de \\ * Corresponding author \\ Keywords: \\ FM-AFM; graphene; 6H-SiC(0001); KPFM; SPM
}

Open Access

\author{
Beilstein J. Nanotechnol. 2012, 3, 179-185. \\ doi:10.3762/bjnano.3.19 \\ Received: 22 November 2011 \\ Accepted: 03 February 2012 \\ Published: 29 February 2012 \\ This article is part of the Thematic Series "Noncontact atomic force \\ microscopy". \\ Guest Editor: U. D. Schwarz
}

(C) 2012 Held et al; licensee Beilstein-Institut.

License and terms: see end of document.

\begin{abstract}
Noncontact atomic force microscopy provides access to several complementary signals, such as topography, damping, and contact potential. The traditional presentation of such data sets in adjacent figures or in colour-coded pseudo-three-dimensional plots gives only a qualitative impression. We introduce two-dimensional histograms for the representation of multichannel NC-AFM data sets in a quantitative fashion. Presentation and analysis are exemplified for topography and contact-potential data for graphene grown epitaxially on $6 \mathrm{H}-\mathrm{SiC}(0001)$, as recorded by Kelvin probe force microscopy in ultrahigh vacuum. Sample preparations by thermal decomposition in ultrahigh vacuum and in an argon atmosphere are compared and the respective growth mechanisms discussed.
\end{abstract}

\section{Introduction}

Graphene grows epitaxially on the $\mathrm{Si}$ face of $6 \mathrm{H}-\mathrm{SiC}(0001)$ by thermal decomposition in vacuum or an inert atmosphere. Recently, fundamental studies have led to an improvement of this process, now allowing for the production of almost wafersize single-layer graphene coverage [1-3]. Understanding the interaction between the substrate and the epitaxial layer during the growth process is crucial for further optimization. Towards this goal, the graphene layer thickness has been determined by various methods including scanning tunnelling microscopy (STM) [4], Raman spectroscopy [5], low-energy electron microscopy [6,7], transmission electron microscopy [8], and atomic force microscopy (AFM) $[9,10]$. AFM also allows the identification of the graphene layer thickness from the local contact potential as determined by means of Kelvin probe force microscopy (KPFM) [11,12]. As a further advantage, KPFM determines step heights more accurately than STM or AFM with constant bias [13] and is therefore employed in this study to investigate the growth mechanisms of graphene on $\operatorname{SiC}(0001)$.

The carbon for graphene growth on $\mathrm{SiC}(0001)$ is obtained from thermal decomposition of the bulk substrate. Heating the sample to temperatures above $1100{ }^{\circ} \mathrm{C}$ leads to Si evaporation and to the formation of carbon-rich reconstructions [3]. At even 
higher temperatures these processes lead to the growth of graphene. A high homogeneity of the graphene coverage was obtained in ultrahigh vacuum by cyclic heating to $1200{ }^{\circ} \mathrm{C}$ [2] and in an argon atmosphere by prolonged heating to $1650{ }^{\circ} \mathrm{C}$ [1]. On the Si face of the $6 \mathrm{H}-\mathrm{SiC}(0001)$ wafers the thickness of the graphene layer is limited to two or three layers. The layer coverage is controlled by the growth temperature rather than by the duration of the heating cycle [14]. The determination of substrate step heights and of related changes in the graphene coverage has already provided interesting insight into the possible growth mechanisms. For example, Charrier et al. observed a preferred step height of one half of a unit cell of $6 \mathrm{H}-\mathrm{SiC}(0001)$ after thermal decomposition [15]. Lauffer et al. correlated these steps with a change in the graphene coverage, based on the observation that one half of a unit cell has almost the same carbon density as one layer of graphene [16].

\section{Experimental}

Noncontact atomic force microscopy (NC-AFM) measurements were performed in ultrahigh vacuum (UHV, $p<2 \cdot 10^{-10}$ mbar) by means of a home-built microscope similar to the one described in [17]. Kelvin probe force microscopy (KPFM) studies were performed in the frequency-modulation mode $[18,19]$. The modulation frequency was set to $1000 \mathrm{~Hz}$ with a bias amplitude of $200 \mathrm{mV}$. Polycrystalline diamondcoated tips (nanosensors) with a typical radius of 20 to $70 \mathrm{~nm}$ were used. Frequencies for the first normal mode of the cantilever were around $100 \mathrm{kHz}$. This choice of cantilever gives the opportunity to perform complementary contact-mode friction and noncontact KPFM experiments on the same surface areas [20].

\section{Graphene grown in UHV}

The substrate material for the study is the Si face of $6 \mathrm{H}-\mathrm{SiC}(0001)$. The unit cell of $6 \mathrm{H}-\mathrm{SiC}$ is composed of six bilayers of $\mathrm{SiC}(0001)$ each with a height of $0.25 \mathrm{~nm}$. Wafers of $6 \mathrm{H}-\mathrm{SiC}(0001)$ were purchased from SiCrystal AG. Polishing scratches were removed by hydrogen etching (grade 5.0, $p=1$ bar, $T=1550{ }^{\circ} \mathrm{C}, t=15 \mathrm{~min}$ ) [1]. After insertion into $\mathrm{UHV}$ and heating to $120^{\circ} \mathrm{C}$ for $10 \mathrm{~h}$ to remove adsorbed water, the surface was imaged by NC-AFM (Figure 1a). Flat terraces with a typical width of $500 \mathrm{~nm}$ were found. The surface of terraces is covered with irregular mounds of up to $0.5 \mathrm{~nm}$ in height. Smaller depressed islands decorate the steps between terraces (see white arrow in Figure 1a). The steps between terraces have a typical height of $1.5 \mathrm{~nm}$, whereas the smaller steps towards the depressed islands have a height of roughly $0.25 \mathrm{~nm}$ (Table 1). The step heights match the height of the $\mathrm{SiC}$

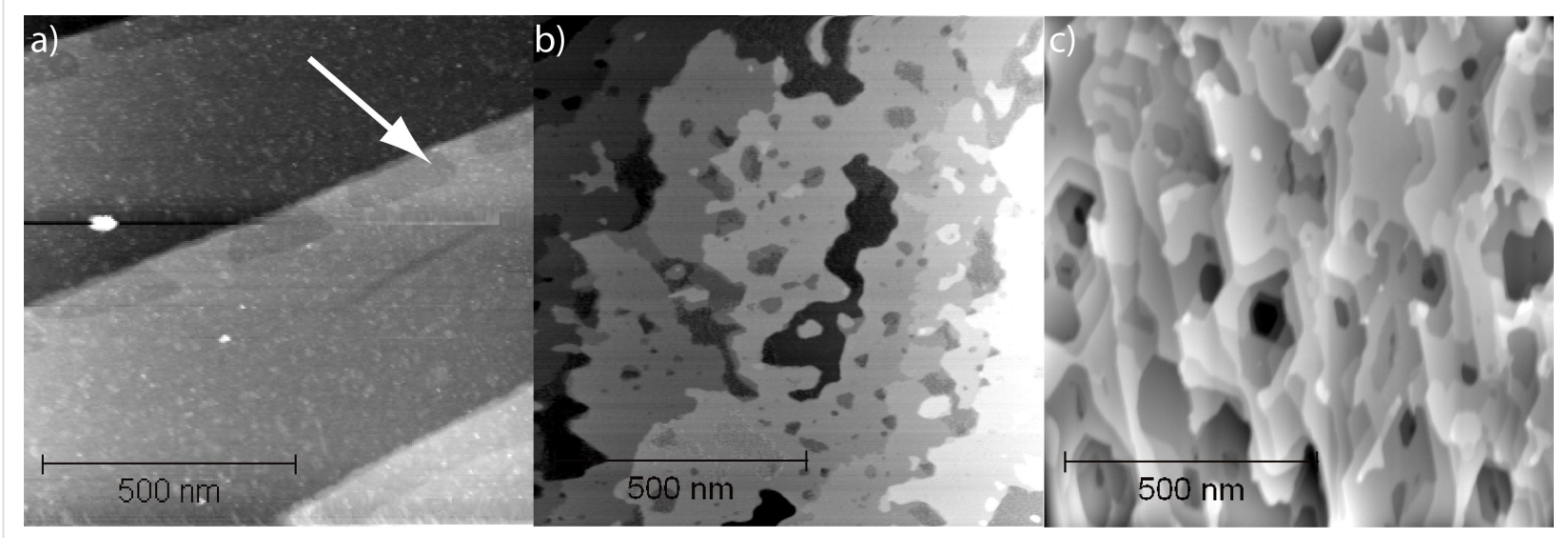

Figure 1: Topography images of the $\mathrm{SiC}(0001)$ sample (a) before annealing, (b) after oxide removal at $1000{ }^{\circ} \mathrm{C}$, and (c) after graphene growth at $1300^{\circ} \mathrm{C}$. Step heights in (a) are $1.5 \mathrm{~nm}$ between the large terraces and $0.25 \mathrm{~nm}$ towards the small depressed islands (indicated by the arrow). Step heights in (b) are $0.25,0.50$ and $0.75 \mathrm{~nm}$, evenly distributed. Step heights in (c) vary from $0.09 \mathrm{~nm}$ to $0.75 \mathrm{~nm}$.

Table 1: Table of different step heights found before and after graphenization of $6 \mathrm{H}-\mathrm{SiC}(1000)$. Dominant step heights are underlined. After graphenization the substrate step heights formed as multiples of the $\mathrm{SiC}(0001)$ bilayer height of $0.25 \mathrm{~nm}$ may vary by the graphene thickness of $0.33 \mathrm{~nm}$.

\begin{tabular}{lll} 
Substrate & Step heights found & Figure \\
\hline Wafer SiC as received & $\underline{1.5}$ and $0.25 \mathrm{~nm}$ & Figure 1a \\
Graphenized in argon & $0,0.25,0.5,0.75,1, \ldots, 2 \mathrm{~nm}$ & Figure 2a, Figure 3a, Figure 4b \\
& $\pm 0.33 \mathrm{~nm}$ for each of the above. & \\
Wafer SiC heated to $1000{ }^{\circ} \mathrm{C}$ & $0.25,0.50,0.75 \mathrm{~nm}$ & Figure 1b \\
Graphenized in UHV & $0.75 \mathrm{~nm} \pm 0.33 \mathrm{~nm}$ & Figure 1c, Figure 2a, Figure 4a
\end{tabular}


unit cell of $1.52 \mathrm{~nm}$ [21] and the SiC bilayer height of $1.52 \mathrm{~nm} / 6=0.253 \mathrm{~nm}$, respectively.

The surface oxide was removed in UHV by direct-current heating $\left(T=1000{ }^{\circ} \mathrm{C}, t=6 \mathrm{~min}\right)$ [3]. The temperature was determined with an infrared pyrometer adjusted to an emissivity of 0.9 . This oxide removal technique is known to change the $\mathrm{SiC}$ surface stoichiometry, as the oxide layer is removed by evaporation of $\mathrm{SiO}$ gas. Overall, the surface structure remains the same upon oxide removal (Figure 2b). The width of the large terraces is slightly reduced and a number of smaller and larger pits and islands with lateral extensions of only a few nanometers up to hundreds of nanometers are found. Except for a few remaining rough spots the surface is now atomically smooth. Step heights between the smooth terraces are mostly $0.25 \mathrm{~nm}, 0.5 \mathrm{~nm}$ and $0.75 \mathrm{~nm}$, which again correspond to multiples of the $\mathrm{SiC}(0001)$ bilayer height (Table 1). The step height between rough spots and adjacent smooth terraces was found to be approximately $0.17 \mathrm{~nm}$ in good agreement with previous studies[10].

Graphene was grown by first heating the sample to $1000{ }^{\circ} \mathrm{C}$ for 6 min to remove contaminants and also to reduce the pressure burst during the subsequent graphenization step of heating to $1300{ }^{\circ} \mathrm{C}$ for $30 \mathrm{~s}$ [3]. This treatment changes the topography significantly (Figure 1c). The largest atomically flat areas now have a lateral extension of only $100 \mathrm{~nm}$. The sample is covered with small pits of hexagonal shape. A large variety of step heights is found (Table 1).

\section{Graphene grown in an argon atmosphere}

The same starting material and sample preparation, i.e., wafer manufacturer, polishing, and hydrogen etching, were used for the graphenization in an argon atmosphere at $1650^{\circ} \mathrm{C}$ following the procedure describe in [1]. After graphenization the sample was introduced into the UHV chamber and heated for $10 \mathrm{~h}$ at $120{ }^{\circ} \mathrm{C}$ in order to remove adsorbed water.

A direct comparison of samples prepared in UHV and in an argon atmosphere reveals huge differences in the surface topography (Figure $2 \mathrm{a}$ and Figure $2 \mathrm{~b}$ ). While the sample prepared in UHV exhibits the pitted structure described above, the sample prepared in an argon atmosphere shows only a few straight step bunches every several microns.

\section{Results}

KPFM measurements reveal variations in the graphene coverage as contributing to the different step heights observed. Figure 3 shows a typical step structure for a sample prepared in an argon atmosphere. Of the two topographic steps (Figure 3a) only one coincides with a change in contact potential (Figure $3 b$ ). The underlying surface structure is analyzed in Figure $3 \mathrm{c}$ and represented in an atomic ball-and-stick scheme in Figure $3 \mathrm{~d}$. The left step is a substrate step of three bilayers of $\mathrm{SiC}$ with a height of $0.75 \mathrm{~nm}$, indicated by the three blue blocks representing the bilayers. The right step is a substrate bilayer step combined with a change in graphene coverage from single to double layer. The resulting topographic step height is $0.09 \mathrm{~nm}$, the change in contact potential $130 \mathrm{mV}$. Such analysis is supported by the fact that steps with a height that is a multiple of the SiC bilayer height never coincide with a change in contact potential. The interface layer introduced in Figure $3 \mathrm{c}$ has been reported as a graphitic layer covalently bound to the $\mathrm{SiC}$ substrate $[4,16]$. While its influence on the electronic structure and contact potential is under discussion, it has no influence on the step heights between graphene-covered terraces.

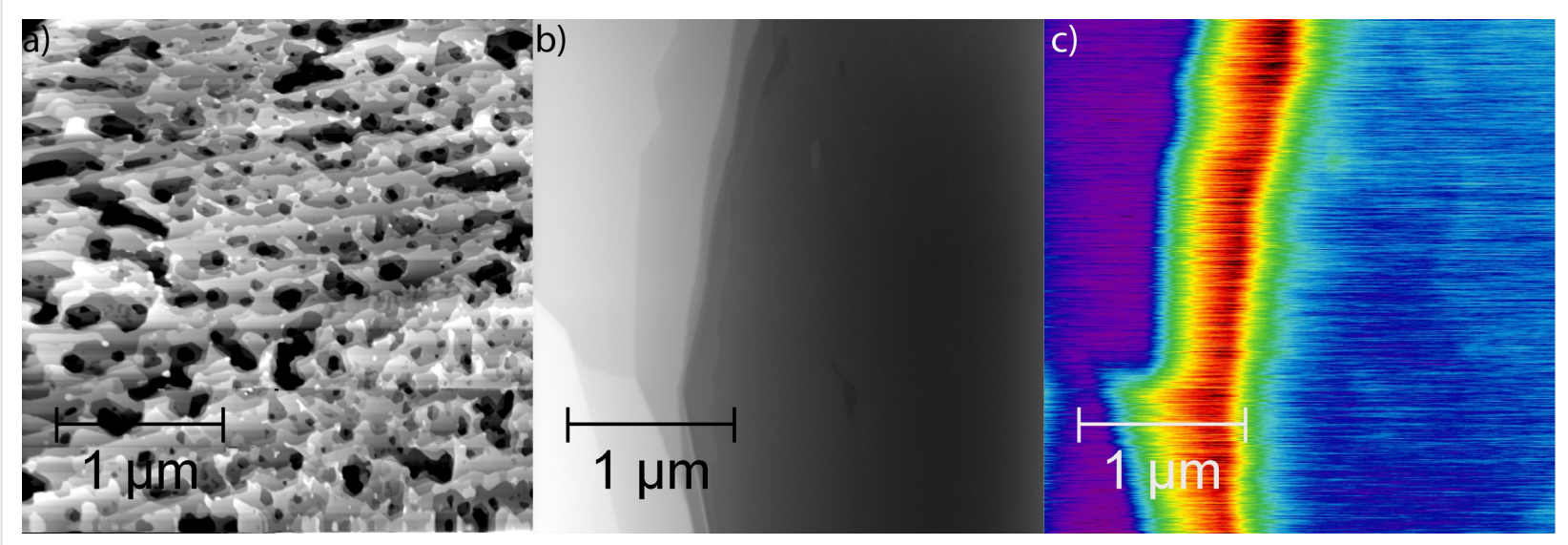

Figure 2: Topography images of graphene layers epitaxially grown on $\mathrm{SiC}(0001)$; (a) preparation in UHV, (b) preparation in an argon atmosphere. Step heights in (a) vary from $0.09 \mathrm{~nm}$ up to $0.75 \mathrm{~nm}$. The total height of the step bunch in (b) is $3.25 \mathrm{~nm}$. The contrast in the contact potential in (c) was recorded simultaneously with the topography in (b). Blue areas indicate single-layer graphene; red areas with $130 \mathrm{mV}$ higher contact potential indicate double-layer graphene. 

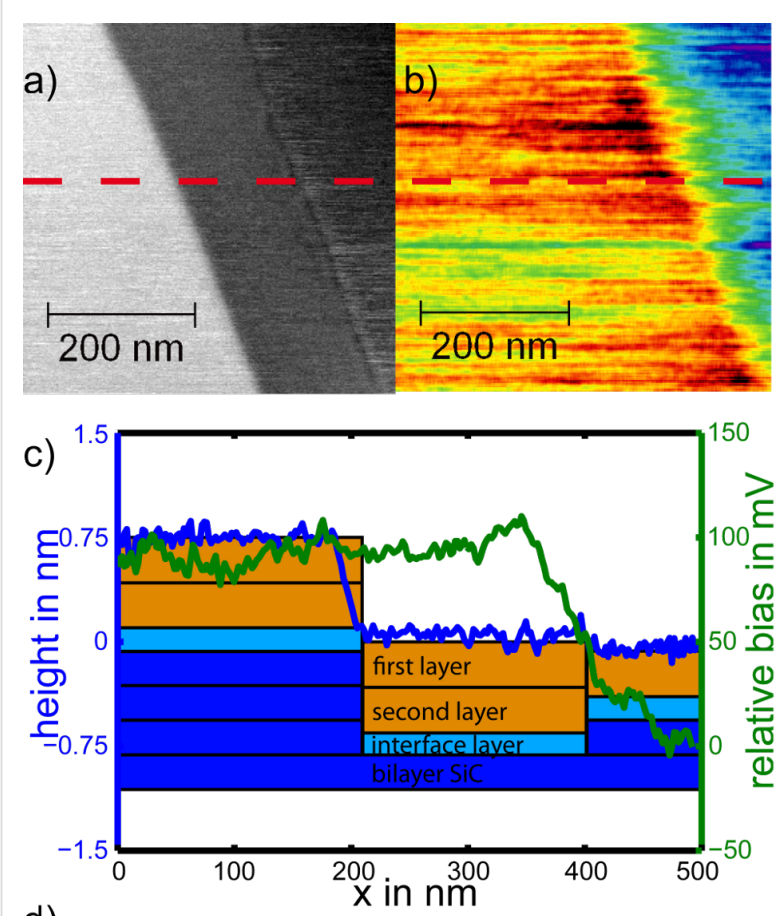

d)

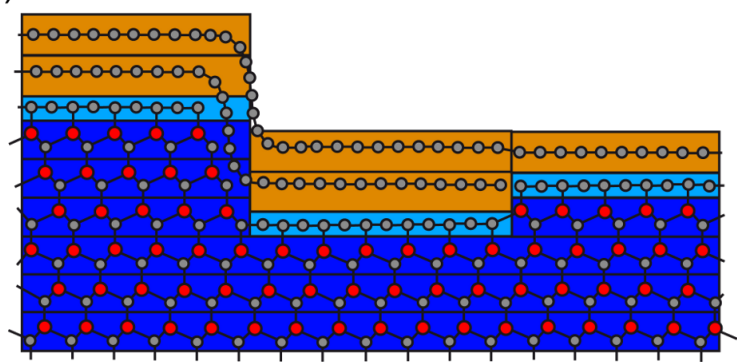

Figure 3: (a) Topographic image showing two steps found typically on samples prepared in an argon atmosphere. (b) Corresponding image of the contact potential difference. Note that only the small step in (a) coincides with a shift in contact potential. (c) Topography (blue) and contact-potential (green) profiles taken along the dashed line in (a). Underlying is a schematic illustration of the corresponding substrate composition. Different layers are drawn to their corresponding step height as $\mathrm{SiC}(0001)$ bilayer $(0.25 \mathrm{~nm}$, blue), interfacial layer (unknown height, light blue), and graphene layer (0.33 nm, orange). (d) Schematic atomic model of the surface structure, showing SiC bilayers, the carbon-rich interface layer, and single- and double-layer graphene.

Rendering the data sets into a pseudo-three-dimensional representation provides an intuitive understanding of the structure and composition of the sample [22]. Figure 4a shows results for a sample prepared in UHV. The topography data is rendered and overlayed with a colour scale representing the local contact potential. Most parts of the sample show a bluish colour indicating single-layer graphene coverage. Some smaller terraces exhibit a higher contact potential represented in red, which indicates double-layer graphene. Double-layer graphene spots are regularly observed to grow over a $\mathrm{SiC}$ bilayer substrate step. No change in contact potential is observed without a corresponding

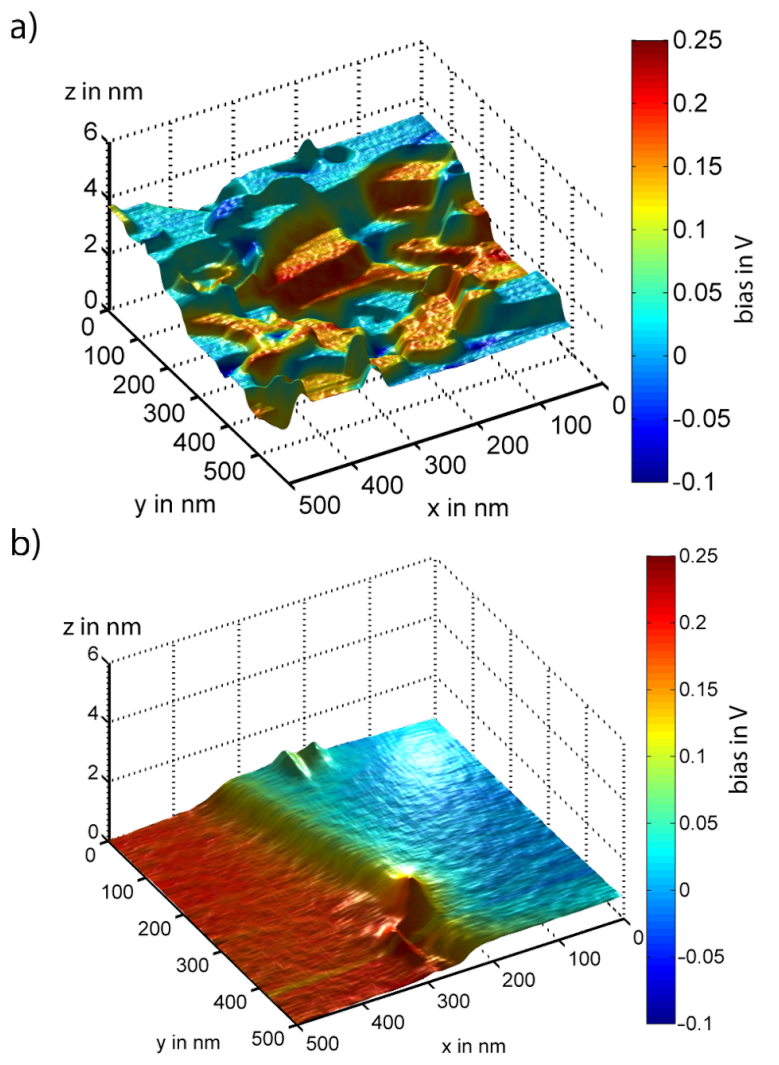

Figure 4: Rendered images of graphene layers on $\mathrm{SiC}(0001)$ prepared in (a) UHV and (b) an argon atmosphere. The colour represents the local contact potential. Bluish colour indicates single-layer graphene, reddish colour double-layer graphene.

change in step height. The much simpler surface structure of samples prepared in an argon atmosphere is demonstrated in Figure $4 b$. The identification of surface areas such as the one in Figure $4 \mathrm{~b}$ by KPFM allows subsequent experiments to be aimed at a direct comparison between single and double layer graphene, for example, in friction experiments.

While this visualization method allows for a quick identification of the surface structure, we will now introduce two-dimensional histograms as a complementary data representation. These histograms are very useful for a quantitative analysis of the complex structures of samples prepared in UHV.

Histograms represent the distribution of values in a given data set. Here we are using two-dimensional histograms to represent the data contained in multichannel NC-AFM frames. Several signal values are assigned to each pixel of a scanned frame, e.g., topography and contact-potential values. Using topography and contact potential as axes of a two-dimensional scatter plot, the frequency of occurrence of each pair of topography and contact- 
potential values is represented by a colour scheme. In this way, topography and contact potential can be graphically correlated while their quantitative values can be directly read from the plot. In order to make two such histograms comparable, topography and contact-potential values are given with respect to the values found in one reference area of the scan frame. A scan frame recorded with 512 lines of 512 pixels provides 262144 data points for this scatter plot, enough for a distinct representation of the relationship between topography and contact potential. Figure 5 shows two-dimensional histograms based on the data sets already presented in the rendered images in Figure 4.

The sample prepared in UHV is analyzed in Figure 5a. Two distinct groups of clustered data points are lined up vertically, reflecting the coverage by single and double-layer graphene. Within each group, a distinct step height of $0.75 \mathrm{~nm}$ is dominant, which corresponds to half the unit cell of $6 \mathrm{H}-\mathrm{SiC}(0001)$. The step height between single- and double-layer graphene terraces is typically $0.42 \mathrm{~nm}$, indicated by green arrows in Figure 5a. It has been suggested that half a unit cell of $\mathrm{SiC}(0001)$ is consumed for the growth of one layer of graphene. This relation suggests itself as the density of carbon atoms is very similar for one half of a unit cell of $\mathrm{SiC}$ and one layer of graphene. The step height of $0.42 \mathrm{~nm}$ is then given as the difference between $0.75 \mathrm{~nm}$ for half a unit cell and $0.33 \mathrm{~nm}$ for the height of one layer of graphene.

The sample prepared in an argon atmosphere is analyzed in Figure 5b, its structure with wide terraces and few steps is reflected in the observation of only two narrow clusters of data points in the histogram. The two groups correspond to a height difference of $0.64 \mathrm{~nm}$, i.e., about $0.33 \mathrm{~nm}$ less than four $\mathrm{SiC}(0001)$ bilayers, which is again the step height of the graphene layer. Therefore we conclude that the lower terrace is depressed by four SiC bilayers but is covered by one additional graphene layer compared to the upper terrace. The extra SiC bilayer decomposed for the structure in Figure $5 \mathrm{~b}$ as compared to Figure $5 \mathrm{a}$ is indicated by the grey arrow.

\section{Discussion}

The results described above shed light on the growth mechanism of graphene on the $\mathrm{Si}$ face of $6 \mathrm{H}-\mathrm{SiC}(0001)$. After oxide removal at $1000{ }^{\circ} \mathrm{C}$ in $\mathrm{UHV}$, the step heights vary between one, two and three bilayers of the $\operatorname{SiC}(0001)$ structure. Subsequent graphenization at $1300{ }^{\circ} \mathrm{C}$ in UHV results in a preferred step height of three bilayers of $\mathrm{SiC}(0001)$. Two mechanisms leading a)

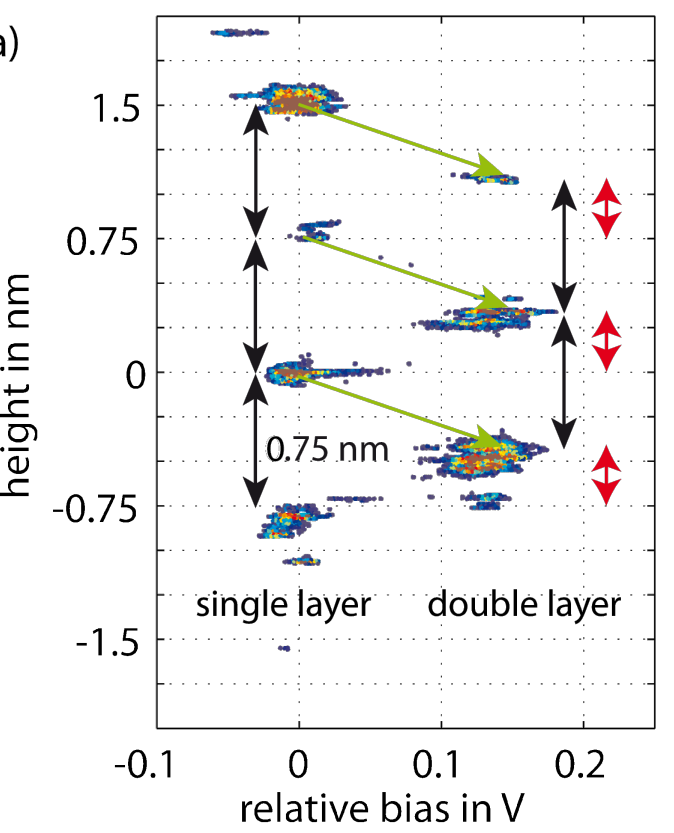

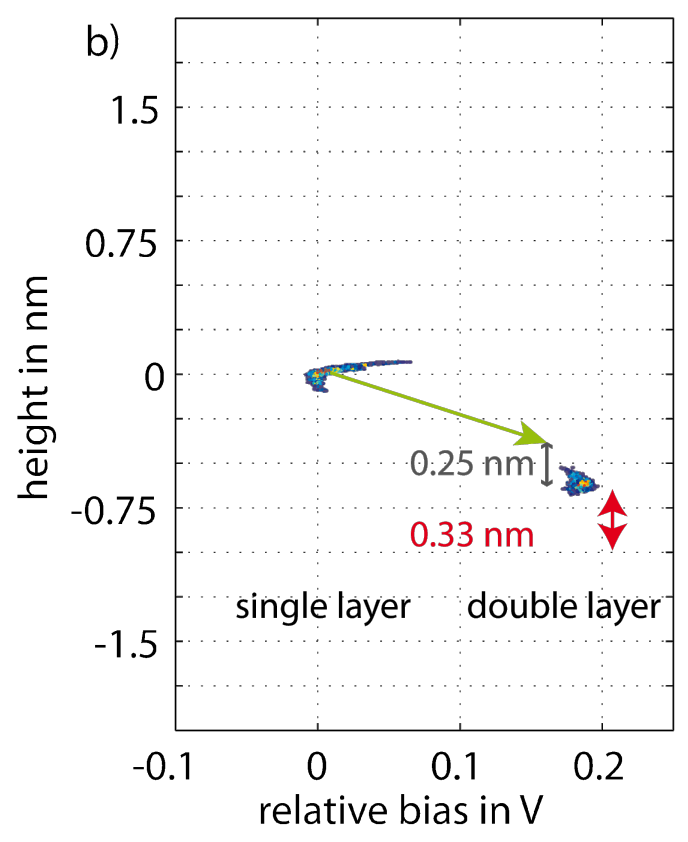

Figure 5: Two-dimensional histograms based on the data set for the rendered images in Figure 4. The colour scheme represents the number of data couples that fall into the respective topography and contact-potential bin; (a) sample prepared in UHV, (b) in an argon atmosphere. Black arrows indicate a height difference of $0.75 \mathrm{~nm}$, equal to half a unit cell of $6 \mathrm{H}-\mathrm{SiC}(0001)$, and the grey arrow a height of $0.25 \mathrm{~nm}$, equal to one bilayer of $\mathrm{SiC}(0001)$. Red arrows indicate the step height of $0.33 \mathrm{~nm}$ corresponding to one graphene layer. Green arrows indicate a suggested graphene growth process, in which three $\mathrm{SiC}$ bilayers are consumed to produce one single graphene layer. Points with less than 5 counts are left transparent to enhance readability of the graph. The colour scale ranges from 5 (blue) to 70 (red) occurrences per $0.01 \mathrm{~nm}$ and $1.75 \mathrm{mV}$. 
to this step height have been suggested. As discussed above, a little more than three bilayers $\mathrm{SiC}(0001)$ provide the carbon atoms required to form one graphene sheet [3]. This simple stochiometric argument is supported by our experimental results, as all spots for single-layer graphene coverage are connected to double-layer graphene spots by the corresponding green arrows in Figure 5. The contact potential difference between single- and double-layer graphene is always found to be close to $130 \mathrm{mV}$.

However, the stochiometric argument does not explain the preferred step height of $0.75 \mathrm{~nm}$ between single-layer graphene areas or between double-layer graphene areas. Hupalo et al. [2] have concluded that different $\mathrm{SiC}$ bilayers within the $\mathrm{SiC}(0001)$ unit cell have different $\mathrm{Si}$ evaporation rates, i.e., the first bilayer of each half unit cell evaporates fastest, followed by the second bilayer, whereas every third bilayer exhibits a low evaporation rate. In Figure 5a all height differences fit multiples of three SiC bilayers. Double layer graphene areas are shifted in height by exactly $0.33 \mathrm{~nm}$, i.e., the thickness of one graphene layer. Therefore, single and double layers of graphene have grown on terraces defined by half unit cells of the $6 \mathrm{H}-\mathrm{SiC}(0001)$ structure. Terraces not following this rule were found rarely, supporting the suggestion of Hupalo et al. for a mechanism of graphene growth in UHV.

Samples prepared in an argon atmosphere differ significantly in step structure. Atomically flat terraces extend over several microns. They are separated by bunches of steps reaching heights of up to $10 \mathrm{~nm}$. The steps have heights that correspond to multiples of a bilayer of $\mathrm{SiC}(0001)$, varying from single up to seven SiC bilayers.

These results indicate that the mechanism described for growth in UHV is not the dominant mechanism for the step structure formation upon growth in argon. The terraces found after graphenization in argon are larger than those found on the starting material, excluding a simple carbon-maintaining transformation of the sample. Furthermore, step heights between the large terraces do not match the height of the half unit cell. The differences may be explained by enhanced diffusion at the elevated temperature of $1650{ }^{\circ} \mathrm{C}$ used for the preparation in argon as compared to $1300{ }^{\circ} \mathrm{C}$ for the preparation in UHV. Several studies have shown that the diffusion of carbon and silicon atoms differs significantly for the two temperatures, for which absolute values are still under discussion [23-25]. Diffusion of carbon atoms from areas with carbon excess to carbondepleted areas appears to be a reasonable mechanism for the formation of larger terraces. Future models of the effect of diffusion will have to take into account the preferred nucleation of double-layer graphene at step bunches.
Samples prepared in argon show an interesting deviation of the contact potential difference between single and double layer graphene from the average value of $130 \mathrm{mV}$. Terraces that are separated by steps with a height other than a half unit cell of $\mathrm{SiC}(0001)$ exhibit contact potential differences of $130 \pm 50 \mathrm{mV}$, examples are presented in Figure $2 \mathrm{c}$ and Figure $5 \mathrm{~b}$. We found no predictable relation between step height and contact potential difference in the available data. The origin of these deviations is not clear at present, but differences in the interface layer between graphene and $\mathrm{SiC}$ at different stacking positions within the unit cell are plausible candidates to explain the variations in contact potential. These differences could express themselves as a variation of the surface reconstruction (e.g., $(5 \times 5),(6 \times 6)$ versus $\left.(6 \sqrt{3} \times 6 \sqrt{3}) \mathrm{R} 30^{\circ}\right)$ of the interface layer [4].

Finally, we add a few comments on the data quality in the twodimensional histograms. The contact potential signal recorded in KPFM shows only a little noise and drift, and can be directly processed in the form of histograms. In contrast, the topography signal needs to be processed to correct for the effects of drift, piezo creep, and piezo hysteresis [26]. The goal, and the justification, for processing is to obtain a minimal curvature of atomically flat terraces. Most NC-AFM operating in UHV do not offer the opportunity to linearize the piezo actuators in a closed-loop scheme. However, for ambient conditions such linearized instruments are commercially available and provide suitable input data for two-dimensional histograms, in particular when the lift-mode KPFM is used [27].

\section{Acknowledgements}

We thank E. Arzt for continuous support of this project. This work was supported by the Deutsche Forschungsgemeinschaft within the European Science Foundation project FANAS.

\section{References}

1. Emtsev, K. V.; Bostwick, A.; Horn, K.; Jobst, J.; Kellogg, G. L.; Ley, L.; McChesney, J. L.; Ohta, T.; Reshanov, S. A.; Röhrl, J.; Rotenberg, E.; Schmid, A. K.; Waldmann, D.; Weber, H. B.; Seyller, T. Nat. Mater. 2009, 8, 203-207. doi:10.1038/nmat2382

2. Hupalo, M.; Conrad, E. H.; Tringides, M. C. Phys. Rev. B 2009, 80, 041401. doi:10.1103/PhysRevB.80.041401

3. Hass, J.; de Heer, W. A.; Conrad, E. H. J. Phys.: Condens. Matter 2008, 20, 323202. doi:10.1088/0953-8984/20/32/323202

4. Riedl, C.; Starke, U.; Bernhardt, J.; Franke, M.; Heinz, K. Phys. Rev. B 2007, 76, 245406. doi:10.1103/PhysRevB.76.245406

5. Shivaraman, S.; Chandrashekhar, M.; Boeckl, J. J.; Spencer, M. G. J. Electron. Mater. 2009, 38, 725-730. doi:10.1007/s11664-009-0803-6

6. Hibino, H.; Kageshima, H.; Maeda, F.; Nagase, M.; Kobayashi, Y.; Yamaguchi, H. Phys. Rev. B 2008, 77, 075413. doi:10.1103/PhysRevB.77.075413

7. Ohta, T.; El Gabaly, F.; Bostwick, A.; McChesney, J. L.; Emtsev, K. V.; Schmid, A. K.; Seyller, T.; Horn, K.; Rotenberg, E. New J. Phys. 2008, 10, 023034. doi:10.1088/1367-2630/10/2/023034 
8. Norimatsu, W.; Kusunoki, M. Physica E 2010, 42, 691-694. doi:10.1016/j.physe.2009.11.151

9. Filleter, T.; Bennewitz, R. Phys. Rev. B 2010, 81, 155412. doi:10.1103/PhysRevB.81.155412

10. Vesapuisto, E.; Kim, W.; Novikov, S.; Lipsanen, H.; Kuivalainen, P. Phys. Status Solidi B 2011, 248, 1908-1914. doi:10.1002/pssb.201046368

11. Curtin, A. E.; Fuhrer, M. S.; Tedesco, J. L.; Meyers-Ward, R. L.; Eddy, C. R., Jr.; Gaskill, D. K. Appl. Phys. Lett. 2011, 98, 243111. doi:10.1063/1.3595360

12. Filleter, T.; Emtsev, K. V.; Seyller, T.; Bennewitz, R. Appl. Phys. Lett. 2008, 93, 133117. doi:10.1063/1.2993341

13. Sadewasser, S.; Lux-Steiner, M. C. Phys. Rev. Lett. 2003, 91, 266101. doi:10.1103/PhysRevLett.91.266101

14. Hass, J.; Feng, R.; Li, T.; Li, X.; Zong, Z.; de Heer, W. A.; First, P. N.; Conrad, E. H.; Jeffrey, C. A.; Berger, C. Appl. Phys. Lett. 2006, 89, 143106. doi:10.1063/1.2358299

15. Charrier, A.; Coati, A.; Argunova, T.; Thibaudau, F.; Garreau, Y.; Pinchaux, R.; Forbeaux, I.; Debever, J.-M.; Sauvage-Simkin, M.; Themlin, J.-M. J. Appl. Phys. 2002, 92, 2479-2484. doi:10.1063/1.1498962

16. Lauffer, P.; Emtsev, K. V.; Graupner, R.; Seyller, T.; Ley, L.; Reshanov, S. A.; Weber, H. B. Phys. Rev. B 2008, 77, 155426. doi:10.1103/PhysRevB.77.155426

17. Howald, L.; Meyer, E.; Lüthi, R.; Haefke, H.; Overney, R.; Rudin, H.; Güntherodt, H.-J. Appl. Phys. Lett. 1993, 63, 117-119. doi:10.1063/1.109732

18. Nonnenmacher, M.; O'Boyle, M. P.; Wickramasinghe, H. K. Appl. Phys. Lett. 1991, 58, 2921-2923. doi:10.1063/1.105227

19. Kitamura, S.; Iwatsuki, M. Appl. Phys. Lett. 1998, 72, 3154-3156. doi:10.1063/1.121577

20. Filleter, T.; McChesney, J. L.; Bostwick, A.; Rotenberg, E.; Emtsev, K. V.; Seyller, T.; Horn, K.; Bennewitz, R. Phys. Rev. Lett. 2009, 102, 086102. doi:10.1103/PhysRevLett.102.086102

21. Bauer, A.; Kräußlich, J.; Dressler, L.; Kuschnerus, P.; Wolf, J.; Goetz, K.; Käckell, P.; Furthmüller, J.; Bechstedt, F. Phys. Rev. B 1998, 57, 2647-2650. doi:10.1103/PhysRevB.57.2647

22. Sadewasser, S.; Glatzel, T.; Rusu, M.; Jäger-Waldau, A.; Lux-Steiner, M. C. Appl. Phys. Lett. 2002, 80, 2979-2981. doi:10.1063/1.1471375

23. Rüschenschmidt, K.; Bracht, H.; Stolwijk, N. A.; Laube, M.; Pensl, G.; Brandes, G. R. J. Appl. Phys. 2004, 96, 1458-1463. doi:10.1063/1.1766101

24. Alfieri, G.; Monakhov, E. V.; Svensson, B. G.; Linnarsson, M. K. J. Appl. Phys. 2005, 98, 043518. doi:10.1063/1.2009816

25. Bedoya-Martínez, B.; Roma, G. Phys. Rev. B 2010, 82, 134115. doi:10.1103/PhysRevB.82.134115

26. Moheimani, R. Rev. Sci. Instrum. 2008, 79, 071101. doi:10.1063/1.2957649

27. Ziegler, D.; Stemmer, A. Nanotechnology 2011, 22, 075501. doi:10.1088/0957-4484/22/7/075501

\section{License and Terms}

This is an Open Access article under the terms of the Creative Commons Attribution License

(http://creativecommons.org/licenses/by/2.0), which permits unrestricted use, distribution, and reproduction in any medium, provided the original work is properly cited.

The license is subject to the Beilstein Journal of Nanotechnology terms and conditions: (http://www.beilstein-journals.org/bjnano)

The definitive version of this article is the electronic one which can be found at:

doi:10.3762/bjnano.3.19 